\title{
Spectrally narrow polarisation conversion in a slow-light photonic crystal waveguide
}

\author{
John Canning \\ j.canning@usyd.edu.au
}

\section{Martin Kristensen}

Nina Skivesen

\section{Cicero Martelli}

\section{Amélie Têtu}

\section{Lars H. Frandsen}

\begin{abstract}
iNANO, ASE and Department of Physics and Astronomy, University of Aarhus, Denmark Interdisciplinary Photonics Laboratories, School of Chemistry, University of Sydney, Australia iNANO, ASE and Department of Physics and Astronomy, University of Aarhus, Denmark
\end{abstract} iNANO, ASE and Department of Physics and Astronomy, University of Aarhus, Denmark

iNANO, ASE and Department of Physics and Astronomy, University of Aarhus, Denmark School of Electrical \& Information Engineering, University of Sydney, Australia

iNANO, ASE and Department of Physics and Astronomy, University of Aarhus, Denmark

A narrow-band ( $3 \mathrm{~dB}$ bandpass $<2 \mathrm{~nm}$ ) transmission notch based on polarisation conversion within a sol photonic crystal waveguide is demonstrated. Signal contrast between quasi- TE and TM eigenstates exceeding $40 \mathrm{~dB}$ is achieved. Further, multiple resonant wavelength coupling between the two eigenstates is also observed. [D0I: 10.2971/jeos.2009.09019]

Keywords: integrated optics, polarisation, sol, silicon, photonics

\section{INTRODUCTION}

Silicon-on-insulator (SOI) technology is the leading platform for integrated photonic and electro-optic circuits [1]. Of these, SOI photonic crystals are seen as a unique approach for practical engineering of highly dispersive compact components for a number of applications where manipulation of an optical field is required [2]-[6]. However, a significant technological challenge remains in fully understanding such properties within even the simplest components. The critical difference in polarisation transmission properties has generally driven a focus on fabricating single polarisation (quasi- TE) photonic chips, although more sophisticated designs enable consideration of both eigenstates [6, 7]. In order to better understand the polarisation properties of such simple crystal structures, we recently carried out a comprehensive evaluation of the polarisation properties of these linear waveguides and showed evidence of significant cross coupling across the entire transmission bands of both quasi- TE and TM (henceforth labeled simply TE and TM) eigenstates [8]. We also reported highly localized polarisation conversion at the TE transmission band edge, principally arising from resonant dispersion and beating demonstrated experimentally and through numerical simulation [8], raising some questions as to the exact definition of this edge. These properties, which were supported by numerical simulation, raise significant questions on the role of dispersion even within single TE polarisation components and necessitate a review of how these components are to be utilized. In this letter, we demonstrate how the dispersion within the TE bandgap can be used to create ultra-narrow polarisation conversion between TE and TM with a signal contrast ra- tio exceeding $40 \mathrm{~dB}$ within the bandgap away from the resonant edge. These results suggest that a more careful evaluation of the bandgap properties of such structures is necessary. By filtering the signal with polarisers narrowband notch filters can be made that are an alternative to cavity-based designs [9] with potential slow light applications. In several cases these filters may solve one of the other outstanding challenges of crystal waveguides, namely the design and fabrication of narrow-band optical add-drop filters.

\section{WAVEGUIDE FABRICATION}

The waveguides were fabricated using a commercial SOI-wafer as platform. Electron-beam lithography (JEOLJBX9300FS) and inductively coupled plasma (ICP) etching were applied to define the photonic crystal (PhC) structure and coupling elements into the $320 \mathrm{~nm}$ top silicon layer.

Figure 1 shows a scanning electron microscope (SEM) image of a typical fabricated crystal structure. Not shown are the adiabatically tapered straight waveguides from $4 \mu \mathrm{m}$ to $0.7 \mu \mathrm{m}$ which allow coupling into and out of the $\mathrm{PhC}$ waveguide. In order to couple light into the structures, lensed end fibres are used to focus light to a $\sim 3 \mu \mathrm{m}$ spot onto the waveguide facet. From the SEM images we measured the triangular lattice constant (pitch), $\Lambda=370 \mathrm{~nm}$, the hole radius $r=125 \pm 5 \mathrm{~nm}$, which gives for this $\mathrm{PhC} r / \Lambda=0.34$, and the Si waveguide thickness, $h=320 \mathrm{~nm}$. The PhC waveguide is created by re- 


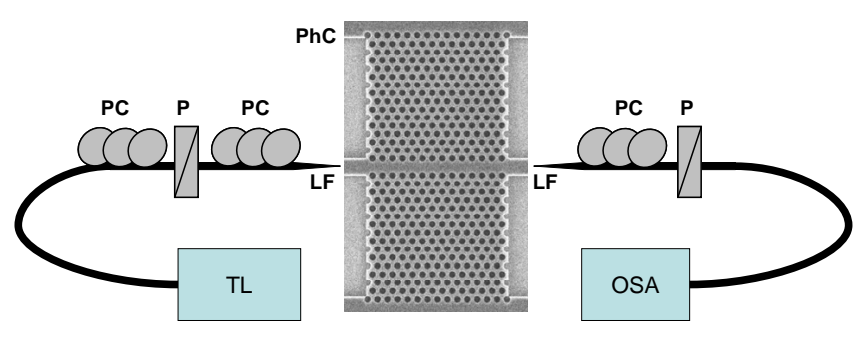

FIG. 1 Schematic of setup employed and SEM image of a $5 \mu \mathrm{m}$ photonic crystal waveguide inserted as the PhC. The actual sample used was $50 \mu \mathrm{m}$ in length but with identical crystal parameters. Also not shown because of size restraints in the imaging process is the SOl tapered input waveguides leading into the structure. $\mathrm{PhC}$-photonic crystal; PC - polarisation controller; P - polariser; LF - lensed fibre; TL - tuneable laser; OSA - optical spectrum analyser.

moving a row of holes along the $\Gamma-\mathrm{K}$ direction. The length of the investigated $\mathrm{PhC}$ waveguides is $50 \mu \mathrm{m}$.

\section{ULTRANARROW FILTER OPERATION AND CHARACTERISATION}

The setup for realising the filter is shown in Figure 1. It consists of the SOI input tapers and crystal waveguide, lensed fibres (LF) for coupling into the SOI tapers and polarisation controllers (PC) and polarisers (P) to allow both full control and monitoring of the polarisation states entering and leaving the device. A tuneable laser (TL) and optical spectrum analyser (OSA) are used to characterise the device.

The premise of the device operation is as follows: from our previous work it was shown that cross coupling between (quasi) TE and TM eigenstates within such photonic crystal waveguides is characterised by a regular beating across the entire telecommunications spectrum and beyond [8]. Such beating is not unexpected since it is identical to coupling between two otherwise orthogonal modes (non-quasi eigenstates) when scattering and/or waveguide asymmetry is present. This can be readily observed at long wavelengths where only TM light propagates but can also be observed within the band edge of the TE transmission pass band. The latter observation is particularly important since it raises practical issues regarding true all-TE only guidance, for example, as well as make uncertain the exact position of the transmission band edge. Such broadband beating is an indicator that the polarisation dispersion is largely material dispersion between air and the silicon across the entire length of the device possibly with some additional contribution from mixing due to excitation of leaky interface states at the ends of the crystal waveguide [10]. This interferogram is primarily determined by the SOI waveguide modal birefringence, $B$, arising from asymmetry of the structure where the phase change, $\phi=2 \pi L B / \lambda$, is over its entire length, $L$, of $2 \mathrm{~mm}$. On the other hand, the $50 \mu \mathrm{m}$ crystal structure gives rise to very large, but narrowband, polarisation dispersion, which is related to the waveguide dispersion by the $Q$ of the lattice structure (i.e. $B_{\text {crystal }} \sim Q B_{\text {waveguide }}$ ) as TE light approaches the TE band edge. The group velocity within a high $Q$ "cavity" determines the slow light properties of the structure.
Although the TM light does experience some dispersion change within the TE bandgap it is small compared to that of the TE light approaching the bandgap defined in principle by the sharp TE transmission edge. This dispersion is significant even before the edge is reached - it is therefore not unreasonable to suppose that phase matching between the polarisation dispersion of the waveguide and the crystal is possible within the TE transmission window for a relatively narrow spectral range because of the large dispersion difference between the two modes involved (TE and TM) [11]. Preliminary observations and numerical simulation have confirmed this is possible [8]. In order to substantially increase the amount of narrow grating-assisted cross coupling between the two polarisation states for novel device purposes, the polarisation input must be optimised carefully. This can be done by initially optimising the bandgap by ensuring only TE light is launched through minimisation of the signal throughput within the TE bandgap. Then the laser and detection system is fixed at one wavelength near the transmission edge and small adjustments are made to the in-coupling polarisation in combination with larger changes to the relative phase of the TE and TM contributions using the polarisation controller at the input. After some adjustment the transmission level starts to drop, and after careful adjustment it is possible to reduce the transmission by more than $40 \mathrm{~dB}$. These results demonstrate that it is possible to manipulate and enhance previous reports on such phenomena [8] to levels that make potentially useful devices.

\section{RESULTS AND DISCUSSION}

Figure 2 shows the optimised situation where a strong resonant notch $(\sim 40 \mathrm{~dB}$ signal contrast between quasi polarisation eigenstates) within the quasi TE transmission window at $1502 \mathrm{~nm}$ is observed. The $3 \mathrm{~dB}$ bandwidth is $<2 \mathrm{~nm}$. The corresponding TM output profile, where the output polariser is rotated $90^{\circ}$, clearly shows that the TE rejected light has been converted to TM as predicted. Also observable in the TM spectra is an additional transmission loss at $\sim 1497.5 \mathrm{~nm}-$ how-

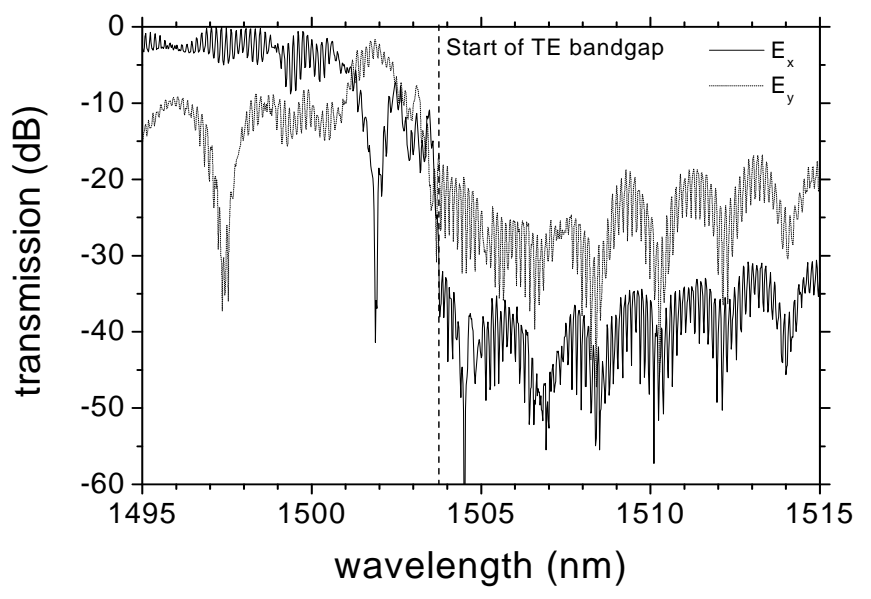

FIC. 2 Transmission spectra of quasi- TE and TM fields through the device. Narrow transmission gap is observed at $1502 \mathrm{~nm}$ for TE light. A corresponding rise in TM signal is seen. 
ever, the signal level is too low to determine whether this has been converted to TE, although this seems likely.

From the interferogram observable at longer wavelengths, where $\Delta \lambda \sim(1.4-2.0) \mathrm{nm}$, we estimate a birefringence of $B_{\text {waveguide }}=\lambda^{2} /(L \Delta \lambda) \sim 0.56$, which is large and arises mainly from the asymmetry of the structure. Depending on the dispersion of the crystal and the exact tuning of phase and amplitude with the input polarisation controller, it is possible to achieve phase matching conditions at other wavelengths than the one shown here. For example, the dispersion at $1502 \mathrm{~nm}$ is not particularly high compared to that approaching the edge - it is reasonable to assume that the measured crystal dispersion of these types of channels [12] for TE eigenstates, is sufficient for conversion of both TE and TM up to the edge and slightly beyond. The signal levels are low for the TM eigenstate in the optimised result above, where TE light beyond the edge is thought to be lost via various mechanisms including back reflection, cladding and lattice modes and some TM conversion. This can be increased by readjusting the input polarisation conditions to see if most of the TE light is coupled into TM whilst still retaining a strong transmission notch. The ability to do this is sensitive to the control over polarisation at both the input and output. Noticeably, we retained high TE transmission below the transmission edge that is the input polariser mostly allows TE through only. Signal transmission was optimised for TE at $1490 \mathrm{~nm}$ whilst at the same time the transmission notch is maintained at $\sim 1502$ nm. Figure 3 shows the raw spectra obtained. A single-peak transmission notch remains although there is significant TM light present. The background signal is nearly uniform - below the band edge it resembles that of Figure 2 (with significant attenuation) whilst at longer wavelengths the signal has increased to comparable levels. The interferogram has disappeared and is replaced instead by a series of decreasing notches (pointed out by arrows on Figure 3), indicative of cross coupling with TE and the formation of additional Fano resonances [13]. The strength of these notches indicates inefficient coupling since TE polarisation propagation is generally

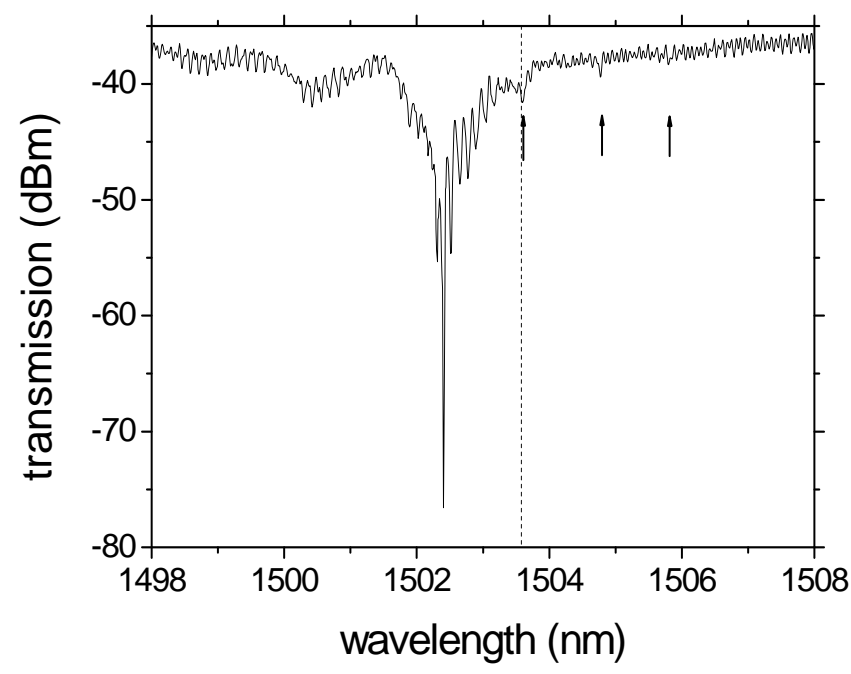

FIG. 3 Transmission resonant notch spectra optimised to have quasi- TE at wavelengths below and quasi- TM at wavelengths above the quasi- TE transmission band edge. Arrows point out additional resonant peaks. forbidden in this region. Despite this, with the significantly smoother transmission signals across the whole spectrum it is now possible to observe multiple wavelength coupling spaced at intervals equal to the interferogram period. A more comprehensive measurement system based on this approach may provide a useful direct characterisation of the dispersion properties of the crystal structures.

The narrow polarisation resonances can be modelled by using the analogy with a simple coupler from classical integrated optics [11] where the two fundamental waveguide modes in adjacent waveguides are replaced with the two polarisation modes TE and TM in the same crystal waveguide. The orthogonality of the ideal eigenstates is broken by the asymmetry of the device enabling analogous beating to occur. However, despite the asymmetry, any analysis needs to consider a larger dispersion difference between the actual polarisation modes than normal modes which tend to have reduced dispersion difference. The resulting equations should be used in the opposite dispersion approximation (very high dispersion difference, similar to the asynchronous solution) compared to the usual case for classical waveguides (similar modes with small dispersion difference). If initially all the light is in quasi-TE, the dispersion is entirely linear, and the propagation losses (and in particular the large difference in propagation loss for TE and TM) are ignored the problem can be solved analytically [11] and one finds for the power coupled to TM:

$$
P_{T M}=\frac{\sin ^{2}\left(\sqrt{v^{2}+\xi^{2}}\right)}{1+\xi^{2} / v^{2}}
$$

where the normalised coupling length, $v$, and the normalised de-phasing parameter, $\xi$, are given by

$$
v=\kappa L \quad ; \quad \xi=\Delta \beta \frac{L}{2}
$$

where $L$ is the length of the waveguide. Since the difference in propagation constants, $\Delta \beta$, (and thereby $\xi$ ) is very large and the coupling strength, $\kappa$, is moderate to large, this gives rise to a narrow sinc function as a function of $L$ or wavelength (analogous equation). Due to the large dispersion and the large difference in loss for TE and TM the coupling profiles will generally be asymmetric with extremely suppressed or even absent side-modes for the sinc function, since the side-modes are more sensitive to de-phasing. In the realistic case there is of course some initial power in TM and a phase difference between the two modes. This is what determines the exact position and depth of the notch in TE (and peak in TM). However, the general equations cannot be solved analytically.

\section{CONCLUSIONS}

By controlling the output polarisation we have been able to demonstrate a novel narrow band-pass filter with more than $40 \mathrm{~dB}$ signal contrast, which exploits the high dispersion available from the quasi- TE eigenstate of the crystal. The asymmetric dispersion that differentiates the transmission bands of $\mathrm{TE}$ and TM, essentially arising from Brewster scattering within the structure [8], potentially allows the generation and tuning of optical Fano-like resonances by adjusting the polarisa- 
tion without directly invoking material nonlinearity. Such resonances can be exploited for slow light generation and applications. Careful phase matching with the TM eigenstate couples the light into the orthogonal polarisation - it is therefore a simple matter to filter out this light using an external polariser on the output. In more sophisticated designs such polarisers can be made onto the chip itself. Alternatively, the coupled light can be taken out and transported into alternative waveguides using appropriately designed couplers [13] or interference regions. Further, the bandwidth of the device can be tailored by tailoring the crystal properties including the length of the structure as well as processing etalon features to further enhance or shape the dispersion profiles. All these features can be substantially enhanced or exploited using various nonlinear effects including both the Kerr and Drude effects [14].

\section{References}

[1] B. Jalali and S. Fathpour, "Silicon Photonics" J. Lightwave Technol. 24 (12), 46100-4615, (2006).

[2] S. Noda, "Recent Progresses and Future Prospects of Two- and Three-Dimensional Photonic Crystals" J. Lightwave Technol. 24 (12), 4554-4567, (2006).

[3] Y. A. Vlasov and S. J. McNab, "Losses in single-mode silicon-oninsulator strip waveguides and bends," Opt. Express 12, 1622-1631 (2004).

[4] L.H. Frandsen, A. Harp $\phi$ th, P.I. Borel, M. Kristensen, J.S. Jensen, and 0 . Sigmund, "Broadband photonic crystal waveguide $60^{\circ}$ bend obtained utilizing topology optimization" Opt. Express 12, 59165921 (2004).

[5] P.I. Borel, B. Bilenberg, L.H. Frandsen, T. Nielsen, J. Fage-Pedersen, A.V. Lavrinenko, J.S. Jensen, 0. Sigmund, and A. Kristensen, “Imprinted silicon-based nanophotonics" Opt. Express 15 (3), 1261-
1266 (2007).

[6] A. Tetu, M. Kristensen, L.H. Frandsen, A. Harp $\phi$ th, P.I Borel, J.S. Jensen, and 0. Sigmund, "Broadband topology-optimized photonic crystal components for both TE and TM polarizations" Opt. Express 13 (21), 8606-8611 (2005).

[7] F. Weng, S. David, X. Checoury, M.E. Kurdi, and P. Boucaud, "Twodimensional photonic crystals with large complete photonic band gaps in both TE and TM polarizations" Opt. Express 16 (16), 1227812289 (2008).

[8] J. Canning, N. Skivesen, M. Kristensen, L.H. Frandsen, A.V. Lavrinenko, C. Martelli, and A. Tetu, "Mapping the broadband polarization properties of linear 2D SOl photonic crystal waveguides" 0 pt. Express 15 (23), 15603-15614 (2007).

[9] B-S. Song, S. Noda, and T. Asano, "Photonic Devices Based on InPlane Hetero Photonic Crystals" Science 300, 1537 (2003).

[10] M. Laroche, F. Marquier, C. Vandenbem, and J.-J. Greffet, "Polarization conversion with a photonic crystal slab" J. Europ. Opt. Soc. Rap. Public. 3, 08038 (2008)

[11] R. Syms and J. Cozens, Optical Guided Waves and Devices (McGrawHill, 1992).

[12] R.S. Jacobsen, A.V. Lavrinenko, L.H. Frandsen, C. Peucheret, B. Zsigri, G. Moulin, J. Fage-Pedersen, and P.I. Borel, “Direct experimental and numerical determination of extremely high group indices in photonic crystal waveguides" 0pt. Express 13, 7861-7871 (2005).

[13] F. van Laere, T. Stomeo, D. Taillaert, G. Roelkens, D. van Thourhout, T.F. Krauss, and R. Baets, "Efficient polarization diversity grating couplers in bonded InP membrane" IEEE Photonic Tech. L. 20, 318320 (2008).

[14] R.S. Jacobsen, K.N. Andersen, P.I. Borel, J. Fage-Pedersen, L.H. Frandsen, 0. Hansen, M. Kristensen, A.V. Lavrinenko, G. Moulin, H. Ou, C. Peucheret, B. Zsigri, and A. Bjarklev, "Strained silicon as a new electro-optic material" Nature 441, 199-202 (2006). 